\title{
Cylindrical Cellular Automata
}

\author{
Erica Jen \\ Theoretical Division, Los Alamos National Laboratory, Los Alamos, NM 87545, USA
}

\begin{abstract}
A one-dimensional cellular automaton with periodic boundary conditions may be viewed as a lattice of sites on a cylinder evolving according to a local interaction rule. A technique is described for finding analytically the set of attractors for such an automaton. Given any one-dimensional automaton rule, a matrix $A$ is defined such that the number of fixed points on an arbitrary cylinder size is given by the trace of $A^{n}$, where the power $n$ depends linearly on the cylinder size. More generally, the number of strings of arbitrary length that appear in limit cycles of any fixed period is found as the solution of a linear recurrence relation derived from the characteristic equation of an associated matrix. The technique thus makes it possible, for any rule, to compute the number of limit cycles of any period on any cylinder size. To illustrate the technique, closed-form expressions are provided for the complete attractor structure of all two-neighbor rules. The analysis of attractors also identifies shifts as a basic mechanism underlying periodic behavior. Every limit cycle can be equivalently defined as a set of strings on which the action of the rule is a shift of size $s / h$; i.e., each string cyclically shifts by $s$ sites in $h$ iterations of the rule. The study of shifts provides detailed information on the structure and number of limit cycles for one-dimensional automata.
\end{abstract}

\section{Introduction}

This paper is concerned with the analysis of one-dimensional cellular automata with periodic boundary conditions. Such an automaton may be viewed as a lattice of sites on a cylinder of specified size $n$ evolving according to a local interaction rule of the form

$$
x_{l}^{t+1}=f\left(x_{i-r}^{t}, \ldots, x_{i}^{t}, \ldots, x_{i+r}^{t}\right), \quad f: Z_{k}^{2 r+1} \rightarrow Z_{k}
$$

together with the condition

$$
x_{i}^{i}=x_{j}^{t}, \quad i \equiv j \bmod n,
$$

for all $i$ and $t$. The values of the sites are restricted to a finite set of integers $Z_{k}=\{0,1, \ldots, k-1\}$. The problem is to study the set of attractors for these automata. Representative questions of interest in this context include the existence of fixed points, the maximum limit cycle period for each cylinder size, and the 
number of distinct attractors for each automata rule. Questions of this type have been studied by Martin, Odlyzko, and Wolfram [1] and by Guan and He [2,3] for rules that are additive and/or linear in the rightmost or leftmost element. Computational results have also been obtained by Wolfram and Peck (see Table 14 in [4]) for all nearest-neighbor rules on cylinders size $\leqq 16$.

An analytical technique is described here for finding the set of attractors for general cylindrical cellular automata. Given any one-dimensional automata rule, a matrix $A$ is defined such that the number of fixed points on an arbitrary cylinder size is given by the trace of $A^{n}$, where the power $n$ depends linearly on the cylinder size. More generally, the number of strings of arbitrary length that appear in limit cycles of any fixed period is obtained as the solution of a linear recurrence relation derived from the characteristic equation of an associated matrix. For any $p$, the technique identifies all cylinder sizes on which a limit cycle period $p$ exists, and thus is dual to the usual approach of fixing $n$ and finding all limit cycles on cylinders size $n$. The end result of the technique, for any rule, is the number of distinct attractors of any period on any cylinder size.

The second major theme of this paper is the identification of shifts as a basic mechanism underlying periodic behavior. Clearly, any automata rule that acts as a shift (in the sense of cyclically permuting the site values in a string) automatically produces limit cycles. Even for general automata rules, however, shifts play a central role in generating limit cycles. In fact, it proves to be surprisingly useful to view any limit cycle for an automation as a set of strings on which the rule acts as a shift of size $s / h$; i.e., each string cyclically shifts by $s$ sites in $h$ iterations of the rule. It is shown here, for example, that for any rule (linear or non-linear) of neighborhood size 2, a string of length $n$ appears in a limit cycle of the rule iff the values of the string satisfy a relation defined using a shift value depending on $n$. The analysis of shifts thus provides detailed information on the structure and number of limit cycles for these automata.

This paper is organized as follows. Section 2 describes the general technique of finding fixed points, and more generally, limit cycles for any automata rule. As an application of the technique, Sect. 3 presents a result pertaining to the uniqueness of fixed points, and the use of cellular automata in pattern recognition, on a cylinder. Section 4 discusses the role of shift mechanisms in the generation of limit cycle behavior. Section 5 builds on the results of the other sections to prove a general theorem on the fundamental role of shifts in generating limit cycle behavior for all two-neighbor rules. Finally, to illustrate the use of the technique introduced here, the Appendix provides closed-form expressions for the complete attractor structure of all two-neighbor rules.

\section{Fixed Points and Limit Cycles for Cylindrical Cellular Automata}

A general technique is presented here for the analysis of fixed-point, and more generally, limit cycle, behavior of cylindrical cellular automata. The essence of the technique is firstly, to identify the "invariant" tuples $\left(x_{-r}, \ldots, x_{r}\right)$ such that the rule assigns

$$
f\left(x_{-r}, \ldots, x_{r}\right)=x_{0}
$$


secondly, to define an "invariance" matrix $A$ with elements equal to 1 for entries representing those tuples satisfying (2.1), and elements equal to 0 otherwise (Eq. (2.2) provides the precise definition of the matrix); and finally, to consider the diagonal elements of $A$ as representing the number of fixed points of the rule on a cylinder size $n$, where $n$ depends linearly on the power. For a fixed period $p$, the technique determines all cylinder sizes on which a limit cycle of period $p$ can exist, and the number of limit cycles of period $p$ on each such cylinder size. In this regard, the technique can be seen as dual to that described in $[8,9]$ (which fixes cylinder size), although the approaches are otherwise quite different.

Definition. An automaton on a cylinder is (ultimately) periodic with period $p$ if there exists a $T \geqq 0$ such that $x_{i}^{t}=x_{i}^{t+p}, 0 \leqq i<n$, for all $t \geqq T$. If $p$ is the smallest positive integer for which the relation is true, then $p$ is the least period of the automaton.

Clearly, any $k$-valued automaton on a cylinder of size $n$ is ultimately periodic with least period $p \leqq k^{n}$. In the remainder of this paper, a string $S=\left\{x_{i}^{t}\right\}$ will be said to appear in a limit cycle of period $p$ if the string is mapped onto itself after $p$ iterations; i.e., the string does not represent a transient in the evolution of the rule.

Definition. Let $S=\left\{x_{i}^{t}, i=0, \ldots, n-1\right\}$ represent a string of length $n$ that appears in a limit cycle period $p$. Then both $S$ and the limit cycle it generates are primitive if $S$ is not spatially periodic of any period $q<n$; i.e., there is no $q<n$ such that

$$
x_{i}^{t}=x_{(i+q) \bmod n}^{t} \text {. }
$$

Notation. For a fixed one-dimensional rule $R$, let

(a) $K_{n}^{(p)}$ denote the number of limit cycles period $p$ of the rule on a cylinder size $n$;

(b) $L_{n}^{(p)}$ denote the number of primitive limit cycles of least period $p$ on a cylinder size $n$

(c) $T_{n}^{(p)}$ denote the total number of (not necessarily primitive) strings of length $n$ that appear in a limit cycle of (not necessarily least) period $p$.

Remark. Let $S=x_{0} \ldots x_{n-1}$ be a string of site values appearing in a limit cycle. If the permuted string $S=x_{i} \ldots x_{n-1} x_{0} \ldots x_{i-1}$ appears in a limit cycle that is distinct from the first (i.e., the two strings never appear in each other's limit cycle), the limit cycles are counted separately.

Lemma 1. With $K_{n}^{(p)}, L_{n}^{(p)}$, and $T_{n}^{(p)}$ defined as above,

$$
K_{n}^{(p)}=\sum_{m \mid n} \sum_{q \mid p} L_{m}^{(q)}
$$

and

$$
T_{n}^{(p)}=\sum_{m \mid n} \sum_{q \mid p} q L_{m}^{(q)}
$$

where " $a \mid b$ " indicates that a divides $b$ exactly.

Proof. The results follow directly from the definitions.

In what follows, it will be assumed that the automata rules being considered are defined on $Z_{2}$. The assumption is made for the purpose of simplifying counting 
arguments; the technique itself is in no way restricted to the case of binary site values.

Now for any rule $f$ of radius $r$, define the $2^{2 r} \times 2^{2 r}$ "invariance" matrix $A$ with elements $a_{i j}$ such that, with $i_{-r} i_{-r+1} \ldots i_{r-1}$ and $j_{-r+1} j_{-r+2} \ldots j_{r}$ representing the right-justified binary forms of $i$ and $j$,

$$
\begin{aligned}
a_{i j} & =1, \quad \text { if } i_{k}=j_{k},-r+1<k \leqq r-1, \text { and } f\left(i_{-r}, j_{-r+1}, j_{-r+2}, \ldots, j_{r}\right)=j_{0}, \\
& =0,
\end{aligned}
$$

The invariance matrix defined above serves the same purpose as the connectivity matrix associated with a graph. Recall that in a connectivity matrix, an element $a_{i j}$ is set equal to 1 iff an edge exists between node $i$ and node $j$. Elements in the matrix raised to a power $n$ then provide a count of paths of length $n$ in the graph that begin at $i$ and end at $j$. For cellular automata, the nodes represent tuples of site values, and the paths can be taken to be spatial (rather than temporal, as might at first be assumed) sequences of these tuples. The matrix contains a " 1 " to indicate that a given value may be appended to a spatial sequence with invariance being preserved, and a " 0 " otherwise.

The first theorem states that the number of fixed points for any rule on a cylinder size $n$ is given by the trace of the corresponding power of the invariance matrix. Corollaries 1.1 and 1.2 then relate this quantity to powers of eigenvalues of the matrix, and thereby to a linear recurrence relation derived from the matrix's characteristic equation. Corollary 1.3 re-expresses the results for the case of limit cycles of arbitrary period. The procedure for computing the number of distinct attractors of any period on any cylinder size is then summarized in the Remark following this set of results.

Theorem 1. For any one-dimensional rule $R$ of neighborhood size $2 r+1$, let $A$ be the invariance matrix defined as in (2.2). Then for $n \geqq 2 r+1, K_{n}^{(1)}$ (or, equivalently, $T_{n}^{(1)}$, the number of fixed points of the rule on a cylinder size $n$, is given by $\operatorname{tr}\left[A^{n-2 r}\right]$.

Proof. An element $a_{i j}^{(1)}$ in the invariance matrix $A=A^{1}$ is non-zero iff the tuple of length $2 r+1$ represented by $\left(i_{-r}, \ldots, j_{r}\right)$ satisfies condition (2.1). The effect of multiplying the invariance matrix $A$ by itself is to set an element $a_{i k}^{(2)}$ in $A^{2}$ equal to the number of paths of length $2 r+2$ beginning with the tuple $\vec{i}=\left(i_{-r}, \ldots, i_{r}\right)$ and ending with the tuple $\vec{k}=\left(k_{-r}, \ldots, k_{r}\right)$ such that each $(2 r+1)$-tuple in the string of site values satisfies condition (2.1). The generalization to $A^{n-2 r}$ is clear. Since the rule is defined on a cylinder, the fixed points of the automaton must begin and end with the same tuple; hence, it is the sum of the diagonal elements (i.e., the trace) that provides the value of $K_{n}^{(1)}=T_{n}^{(1)}$.

Corollary 1.1. Let $\left\{\lambda_{i}, i=1, \ldots, N\right\}$ denote the set of eigenvalues of the invariance matrix $A$. Then for $n \geqq 2 r+1, K_{n}^{(1)}=T_{n}^{(1)}=\sum_{i=1}^{N} \lambda_{i}^{n-2 r}$.

Corollary 1.2. For a rule $R$, let

$$
\lambda^{N}+a_{N-1} \lambda^{N-1}+\cdots+a_{0}=0
$$


represent the characteristic equation of the invariance matrix $A$. Then for $n \geqq N+1$, $K_{n}^{(1)}=T_{n}^{(1)}$ satisfies the linear recurrence relation

$$
T_{n}^{(1)}+a_{N-1} T_{n-1}^{(1)}+\cdots+a_{0} T_{n-N}^{(1)}=0 .
$$

Since any limit cycle of least period $p>1$ can be regarded as a fixed point of the $p^{\text {th }}$ order composition of the rule, the following result is obtained.

Corollary 1.3. Let $A_{p}$ denote the invariance matrix for the $p^{\text {th }}$ order composition of a rule $R$ with eigenvalues $\left\{\lambda_{i}^{(p)}, i=1, \ldots, M\right\}$, and let

$$
\lambda^{M}+a_{M-1}^{(p)} \lambda^{M-1}+\cdots+a_{0}^{(p)}=0
$$

represent the characteristic equation of the invariance matrix $A_{p}$. Then for $n \geqq M+1$,

$$
T_{n}^{(p)}=\sum_{i} \lambda_{i}^{n-2 r p}
$$

and $T_{n}^{(p)}$ satisfies the linear recurrence relation

$$
T_{n}^{(p)}+a_{M-1}^{(p)} T_{n-1}^{(p)}+\cdots+a_{0}^{(p)} T_{n-M}^{(p)}=0 .
$$

Proof. Each primitive string in a limit cycle least period $q$ is a fixed point of the $q^{\text {th }}$ order rule, and hence, for any $p$ with $q \mid p$, a fixed point of the $p^{\text {th }}$ order rule as well. Moreover, if the string is of length $m$, then its concatenations are fixed points on all cylinders of size $n$ where $m \mid n$. Thus, in the trace of the matrix $A_{p}^{n-2 r p}$, each primitive limit cycle is represented by a term equal to its period; i.e.,

$$
\begin{aligned}
\sum_{i} \lambda_{i}^{n-2 r p} & =\sum_{m \mid n} \sum_{q \mid p} q L_{m}^{(q)}, \\
& =T_{n}^{(p)} .
\end{aligned}
$$

Remark. The preceding results provide an algorithm for finding the number of distinct attractors of any period on any cylinder size. To determine $L_{n}^{(p)}$ for all $n$ and some fixed $p$, for example, the procedure is as follows:

(i) Use Eq. (2.3) to find $T_{n}^{(1)}$ for all $n$. Then for any prime $d, L_{d}^{(1)}=T_{d}^{(1)}$.

(ii) From Lemma 1, $L_{n}^{(1)}=T_{n}^{(1)}-\sum_{\left.m\right|^{*} n} L_{m}^{(1)}$, where $\left.m\right|^{*} n$ indicates that $m \mid n$ and $m \neq n$. Thus, $L_{n}^{(1)}$ can be found for any $n$.

(iii) Repeat calculations (i) and (ii) for each period size $q$ with $\left.q\right|^{*} p$ to obtain $L_{n}^{(q)}$ for all $n$. Begin with the smallest value of $q$ and at each step, take the next largest.

(iv) Find $T_{n}^{(p)}$. From Lemma $1, L_{n}^{(p)}=T_{n}^{(p)}-\sum_{\left.m\right|^{*} n} \sum_{\left.q\right|^{*} p} q L_{m}^{(q)}$.

The use of Corollary 1.3 to determine $T_{n}^{(p)}$ becomes increasingly cumbersome unless induction is possible using results for limit cycles of small period; this is done in the Appendix for two-neighbor automata rules. For the present purpose of illustrating the extension from fixed-points to limit cycles of period $p>1$, consider the rule defined by $\{000,010,011,101,110,111\} \rightarrow 0,\{001,100\} \rightarrow 1$ (labelled Rule 18 in $[1,4]$ ), a nonlinear rule. The characteristic equation for $p=1$ (omitting the term consisting of factors of $\lambda$ ) is given by

$$
\lambda-1=0
$$


implying that $\sum_{i} \lambda^{n}=1$ for all $n$, and hence

$$
\begin{aligned}
T_{n}^{(1)} & =1, & & n>0, \\
L_{n}^{(1)} & =1, & & n=1, \\
& =0, & & \text { otherwise. }
\end{aligned}
$$

For $p=2$, the characteristic equation (again omitting factors of $\lambda$ ) is given by

$$
(\lambda-1)\left(\lambda^{6}-\lambda^{2}-2 \lambda-1\right)=0,
$$

implying, from Lemma 1 and Corollary 1.3,

$$
\sum_{m \mid n} L_{m}^{(2)}=\frac{T_{n}^{(2)}-1}{2}, \text { for all } n,
$$

and

$$
T_{n}^{(2)}-1=T_{n-4}^{(2)}+2 T_{n-5}^{(2)}+T_{n-6}^{(2)}-4, \quad n>6
$$

or

$$
T_{n}^{(2)}=T_{n-4}^{(2)}+2 T_{n-5}^{(2)}+T_{n-6}^{(2)}-3, \quad n>6
$$

with initial conditions

$$
T_{1}^{(2)}=T_{2}^{(2)}=T_{3}^{(2)}=1, \quad T_{4}^{(2)}=5, \quad T_{5}^{(2)}=11, \text { and } T_{6}^{(2)}=7,
$$

found by calculating the traces of the appropriate matrices $A_{2}^{n}$. Recurrence relations of this type can be derived for any value of the period $p$.

In what follows, references to fixed points should be understood to generalize to limit-cycle behavior.

Note that in the case of Rule 18 above, the number of limit cycles for $p=2$ increases as a function of cylinder size. By contrast, there are rules for which the number of limit cycles of fixed period is essentially bounded independent of cylinder size. In particular, this feature is true of rules for which the characteristic equation associated with the invariance matrix is of the form

$$
\prod_{i}\left(\lambda^{m_{i}}-1\right)^{\alpha_{i}}=0
$$

for some set of positive integers $m_{i}, \alpha_{i}$. Then Theorem 1 implies (in the case, for example, $p=1$ ) that

$$
\begin{aligned}
T_{n}^{(1)} & =\sum_{i \in M} \alpha_{i} m_{i}, \quad M=\left\{i: m_{i} \mid n\right\} \\
L_{n}^{(1)} & =\alpha_{i} m_{i}, \quad n=m_{i} \\
& =0, \quad \text { otherwise. }
\end{aligned}
$$

In other words, (i) $\alpha_{i} m_{i}$ primitive fixed points exist on cylinders size $m_{i}$; (ii) for any cylinder size $n$ a multiple of $m_{i}$, there are $\alpha_{i} m_{i}$ fixed points that are concatenations of copies of the primitive fixed point; and (iii) no other fixed points exist. Thus, generalizing from the case $p=1$, the number of limit cycles for any period $p$ is bounded above (modulo concatenations as in (ii) above) independent of cylinder size. 
The boundedness of the cylinder size on which a limit cycle can appear is a direct consequence of the fact that the characteristic equation for all matrices $A_{p}$ associated with some rule can be written in the form (2.5). This is always true if the rule is one-to-one in a sense defined in [5]; i.e., if the rule $f$ satisfies either of the following two conditions:

or

(i) $f\left(0, x_{0}, \ldots, x_{2 r}\right) \neq f\left(1, x_{0}, \ldots, x_{2 r}\right)$,

(ii) $f\left(x_{0}, \ldots, x_{2 r}, 0\right) \neq f\left(x_{0}, \ldots, x_{2 r}, 1\right)$,

for every tuple $\left(x_{0}, \ldots, x_{2 r}\right)$, where $r$ is the radius of the rule. If neither of the above two conditions is satisfied (i.e., the rule is two-to-one in the sense of [5]), as is the case for Rule 18, the number of limit cycles of period $p$ can increase as a function of cylinder size $n$.

As an example, consider the nonlinear nearest-neighbor rule defined by

$$
\{000,011,101,111\} \rightarrow 0, \quad\{001,010,100,110\} \rightarrow 1
$$

(labelled Rule 30 in [4]). Then the characteristic equations for $A_{1}, A_{3}$, and $A_{4}$, for example, are given by

$$
\begin{aligned}
(\lambda-1)\left(\lambda^{2}-1\right) & =0, \\
(\lambda-1)\left(\lambda^{2}-1\right)\left(\lambda^{12}-1\right) & =0,
\end{aligned}
$$

and

$$
(\lambda-1)\left(\lambda^{2}-1\right)\left(\lambda^{7}-1\right)^{4}=0
$$

respectively, implying that

$$
L_{1}^{(1)}=1, \quad L_{2}^{(1)}=2, \quad L_{12}^{(3)}=4, \quad L_{7}^{(4)}=7,
$$

and for $p=1,2,3,4$,

$$
L_{n}^{(p)}=0, \quad \text { otherwise }
$$

Thus, for instance, the only primitive limit cycles of least period 3 appear on cylinders size 12 . On a cylinder size 24 , say, the results $(2.7)$ together with Lemma 1 give $K_{24}^{(3)}=7$. The seven attractors exhibiting period 3 behavior include: one fixed point that is a concatenation of 24 copies of the fixed point on the cylinder size 1 (i.e., all 0's); two fixed points that are concatenations of 12 copies of the fixed points on the cylinder size 2 (i.e., 10 and 01); and four limit cycles least period 3 that are concatenations of 2 copies of the limit cycle on the cylinder size 12 (i.e., the string 111110010000 , and the three strings obtained by cyclically permuting the string by one, two, or three sites).

The cylinder sizes for Rule 30 on which primitive limit cycles of period $\leqq 10$ exist, together with the number of limit cycles on each such cylinder, are given in Table 1.

As demonstrated in the Appendix, the technique introduced in this section can be used to obtain, for any two-neighbor automata rule and given parameters $p$ and $n$, the number of limit cycles of period $p$ on cylinder size $n$. Further, these quantities can be generalized, using induction and in some cases the techniques 
Table 1. Primitive limit cycles of least period $p \leqq 10$ and associated shifts for Rules 60 and 30. The notation " $m \times n$ " under the "cylinders" column indicates the existence of $m$ distinct cycles on cylinders size $n$

\begin{tabular}{|c|c|c|c|c|}
\hline \multirow{2}{*}{$\begin{array}{l}\text { Period } \\
p\end{array}$} & \multicolumn{2}{|c|}{ CA 60} & \multicolumn{2}{|l|}{ CA 30} \\
\hline & Cylinders & Shifts & Cylinders & Shifts \\
\hline 1 & $1 \times 1$ & -1 & $1 \times 1,1 \times 2$ & -11 \\
\hline 2 & & & & \\
\hline 3 & $1 \times 3$ & -1 & $4 \times 12$ & 4 \\
\hline 4 & & & $7 \times 7$ & $\frac{7}{7}$ \\
\hline 5 & $3 \times 15$ & -3 & $1 \times 5,3 \times 15,5 \times 25$ & $2,3,10$ \\
\hline 6 & $2 \times 6$ & $-\frac{2}{2}$ & $14 \times 84$ & 14 \\
\hline 7 & $9 \times 7$ & $\frac{7}{7},-2,-4$ & $15 \times 15$ & $\frac{15}{7}$ \\
\hline 8 & & & $1 \times 4,10 \times 80$ & $\frac{1}{2}, 10$ \\
\hline 9 & $28 \times 63$ & $-\frac{1}{7}$ & $5 \times 15,15 \times 135$ & $\frac{5}{3}, 15$ \\
\hline 10 & $24 \times 30$ & $-\frac{6}{2}$ & $18 \times 90,31 \times 155$ & $-\frac{31}{2},-\frac{36}{2}$ \\
\hline
\end{tabular}

for linear cellular automata described in [6], to provide closed-form expressions for the number of limit cycles as a function of $p$ and $n$.

\section{Uniqueness of Fixed Points on a Cylinder}

As an application of the approach described in Sect. 2, this section presents a result pertaining to the uniqueness of fixed points, and the use of cellular automata in pattern recognition on a cylinder. The result is a variant of those contained in [7], and will be proved using the present technique.

The question is to find rules $R$ such that a prescribed string $P=p_{0} \ldots p_{n-1}$ is the unique (modulo concatenations) fixed point of $R$ on all possible cylinders. In [7], it was shown that, given any string $P$ and any $r$, a simple procedure can be used to construct the automata rules of radius $r$ for which $P$ is a fixed point. In general, for any such rule, there will be strings different from $P$ that will also be fixed points. The minimal value $r^{*}$ such that $P$ is the unique fixed point of appropriately defined rules of radius $\geqq r^{*}$ will be shown to be dependent on the length of the "longest self-match" in $P$.

Remark. Uniqueness of $P=p_{0} \ldots p_{n-1}$ as a fixed point is taken here to mean that the only fixed points are strings whose lengths are multiples of $n$, and which consist of concatenations of "complete" copies of $P$. Thus, if the string 1011110 is the unique fixed point of a rule, then the string 10111 on a cylinder size 5 is not; i.e., overlaps are not allowed. The corollary following Theorem 2 can be used to identify the minimal radius rule if overlaps are in fact to be allowed.

For convenience, it will be assumed throughout this section that the rules being considered are of odd neighborhood size.

Definition. Let $P=\left\{p_{0} \ldots p_{n-1}\right\}$ be an arbitrary string. Define $J_{r}$ to be the set of 
tuples $\left(x_{-r}, \ldots, x_{r}\right)$ obtained by setting, for each $i=0, \ldots, n-1$,

$$
x_{j}=p_{(i+j) \bmod n}, \quad j=-r, \ldots, 0, \ldots, r .
$$

Then the rule $R$ of radius $r$ induced by $P$ assigns

$$
x_{-r} \ldots x_{0} \ldots x_{r} \rightarrow x_{0}
$$

for each tuple in $J_{r}$, and

$$
x_{-r} \ldots x_{0} \ldots x_{r} \rightarrow x_{0}^{\prime},
$$

where $x_{0}^{\prime} \neq x_{0}$, for all tuples $\left(x_{-r}, \ldots, x_{r}\right) \notin J_{r}$.

Clearly, the string $\left\{p_{0} \ldots p_{n-1}\right\}$ is the unique fixed point of the induced rule of neighborhood size $n$.

The next result provides a less obvious upper bound on the neighborhood size needed to guarantee uniqueness of the fixed point. Essentially, it will be shown that ambiguities are resolved, and uniqueness attained, by using a neighborhood size greater than the length of the longest tuple that appears twice in the fixed-point.

Definition. A string $P=\left\{p_{0} \ldots p_{n-1}\right\}$ possesses a self-match of length $m>0$ if there appear in the string two identical tuples $\left(p_{i}, \ldots, p_{i+m-1}\right)$ and $\left(p_{j}, \ldots, p_{j+m-1}\right)$ with $i \neq j$ and with the indices taken modulo $n$.

Now define for any string $P$

$$
\begin{aligned}
r^{*} & =\frac{m+1}{2}, \quad m \text { odd }, \\
& =\frac{m+2}{2}, \quad m \text { even, }
\end{aligned}
$$

where $m$ is the length of the longest self-match in $P$.

Theorem 2. A primitive string $P=\left\{p_{0} \ldots p_{n-1}\right\}$ is the unique fixed point on all possible cylinders for a rule induced by $P$ iff the rule is of radius $r \geqq r^{*}$, where $r^{*}$ is defined in (3.1).

Proof. Let $R$ be a rule induced by $P$ with radius $r \geqq r^{*}$, and let $A$ be the invariance matrix associated with $R$. Two elements $a_{i j}$ and $a_{i k}$ cannot both be equal to 1 , since that would imply that the tuple $\vec{i}=\left(i_{-r}, \ldots, i_{r}\right)$ appears twice in $P$. Similarly, no column in $A$ contains more than one non-zero element. Moreover, since $P$ is of length $n$, there is a total of exactly $n$ 1's in $A$. Consequently, the characteristic equation for $A$ (omitting the term consisting of factors of $\lambda$ ) is given by $\lambda^{n}-1=0$, and hence $K_{n}^{(1)}=L_{n}^{(1)}=1$, and $L_{m}^{(1)}=0$ for all $m \neq n$, implying that $P$ is the unique fixed point (modulo concatenations of copies of $P$ ) on all possible cylinders.

Now show that any induced rule of radius $<r^{*}$ has a fixed point different from $P$. Let $m$ be the length of the longest self-match in $P$. Assume that $P$ has the form

$$
\begin{aligned}
P & =p_{0} \ldots p_{n-1} \\
& =q_{0} q_{1} \ldots q_{m-1} p_{m} \ldots p_{m+a} q_{0} q_{1} \ldots q_{m-1} p_{2 m+a+1} \ldots p_{n-1},
\end{aligned}
$$

where $\vec{q}=\left(q_{0} q_{1} \ldots q_{m-1}\right)$ represents the longest tuple that appears twice in $P$. (The 
argument is the same if $\vec{q}$ overlaps with itself.) Then the string

$$
P^{\prime}=q_{0} q_{1} \ldots q_{m-1} p_{m} \ldots p_{m+a}
$$

is a fixed point for any rule induced by $P$ of radius $<r^{*}$.

The next corollary provides a generalization of the above theorem to the question of defining a rule for which two or more specified strings are to be the unique fixed points. Given a set of multiple strings, the induced rule is defined as for the case of one string, where now the set $J_{r}$ contains all $2 r+1$ tuples appearing in any member of the set.

Corollary 2. Let $\left\{P_{j}\right\}$ be a set of arbitrarily chosen primitive strings of possibly unequal lengths $n_{j}$. For each $P_{j}$, let $r_{j}^{*}$ be the radius defined in (3.1) for that string. Set $N=\operatorname{lcm} n_{j}$, and for each $P_{j}$ construct the string $\bar{P}_{j}$ of length $N$ consisting of (unmixed) concatenations of copies of $\overline{P_{j}}$. Now let $\bar{P}$ be the longest tuple that is common to all strings $\overline{P_{j}}$, and let $\bar{r}$ be the radius defined in (3.1) for $\bar{m}=\operatorname{len}(\bar{P})$. Then for the rule induced by $P_{\text {J }}$ with radius $r \geqq \max \left(r^{*}, \bar{r}\right)$ and with arbitrary initial conditions, the only fixed points are the strings $P_{j}$.

As an example, suppose a rule is to be defined for which the string $P_{1}=101011$ is the unique fixed point on all cylinders. Then the longest self-match in $P_{1}$ is the sub-string 101 of length $m_{1}=3$, and thus from (3.1), $r_{1}=2 . P_{1}$ is therefore the unique fixed point of any rule induced by $P_{1}$ of radius $\geqq 2$. The smallest such rule is that defined by

$$
\{01011,11010\} \rightarrow 0, \quad\{01110,10101,10111,11101\} \rightarrow 1,
$$

with all other tuples $\left(x_{-2}, \ldots, x_{2}\right) \rightarrow \overline{x_{0}}$.

Now suppose that a rule is to be chosen such that the fixed points consist of $P_{1}, P_{2}$, and their concatenations. Find the longest tuple that appears in both $P_{1}$ and a string of length 6 composed of concatenations of $P_{2}$; i.e., 101010. The longest such tuple is 10101 of length $\bar{m}=5$, and thus $\bar{r}=3$. Thus, the given strings are the unique fixed points of the 7-neighbor rule defined by

$$
\begin{aligned}
& \{1010101,1010111,1110101\} \rightarrow 0, \\
& \{0101010,0101110,0111010,1011101,1101011\} \rightarrow 1 .
\end{aligned}
$$

The above corollary can be extended in a straightforward fashion to establish the minimum radius of the rule whose fixed points include, in addition, strings representing mixed concatenations of the given strings $P_{j}$.

\section{Shifts, Cylinder Size, and Limit Cycles}

The analysis of the attractor structure for cylindrical cellular automata uncovers an unexpected feature of their generation of limit cycles: namely, it indicates that shift transformations are the underlying mechanism in this behavior. Clearly, any automaton that acts as a shift (in the sense of cyclically permuting site values on the cylinder) automatically produces limit cycles. Surprisingly, it can be shown that the reverse is also true for what appears to be a wide class of one-dimensional 
rules. In particular, for any two-neighbor rule, a limit cycle can be defined exactly as a set of strings such that the action of the rule on those strings is a shift. Analysis of shifts for a rule then provides detailed information on the structure of its attractors.

To clarify terminology, it should be noted that the shift transformations studied here differ from others in the cellular automata literature. Shifts have appeared in work on the ergodic and information- theoretic aspects of cellular automata, including studies by Hedlund [8] describing cellular automata as continuous maps that commute with a shift map, by Lind [9] on shift-invariant measures and statistical features of shift maps acting on cellular automata sequences, and by Boyle [10] on the relationship between the entropy of a shift and degrees of endomorphisms. In general, these studies consider the composition of one map (a shift) with a second map (an automata rule). This paper describes, instead, the equivalence between automata rules and shifts when restricted to certain subsets of sequences.

Definition. For a given rule $R$ defined on a cylinder size $n$, let $S=$ $\left\{x_{i}^{t}, i=0, \ldots, n-1 ; t=0, \ldots, p-1\right\}$ represent a limit cycle of period $p$. Then the rule acts on $S$ as a shift of size $\sigma=s / h$, with $s, h$ both integers and $h>0$, if

$$
x_{i}^{t}=x_{(i+s) \bmod n}^{t+h}
$$

for all $i$ and $t$; i.e., each string in the limit cycle undergoes a cylical permutation by $s$ sites to the right after $h$ iterations. The shift value $\sigma=s / h$ is fundamental if $h$ is the smallest positive integer for which the relation holds for any $s$.

The following results describe the interplay of limit cycle period, shift size, and cylinder size for automata rules. The theorem deals with two questions: (i) the numerical relationship among period, shift, and cylinder size; and (ii) the identification of a recurrence relation satisfied by any string of values exhibiting shift behavior. To illustrate some of the results of the theorem, Table 1 provides shift and cylinder sizes for limit cycles of period $p \leqq 10$ for Rule 30, defined by (2.6), and for Rule 60, defined by

$$
\{000,001,110,111\} \rightarrow 0, \quad\{010,011,100,101\} \rightarrow 1 .
$$

Theorem 3. Let $R$ be a one-dimensional rule defined on a cylinder size $n$, and suppose there exists for $R$ a primitive limit cycle of least period $p$ for which the rule acts as a shift of value $\sigma=s / h$. Then

(i) if $\sigma$ is fundamental, $p, n$, and $s / h$ satisfy

$$
p=\frac{n h}{\operatorname{gcd}(n,|s|)} .
$$

Otherwise, if the fundamental shift value is given by $\sigma_{1}=s_{1} / h_{1}$, then

$$
p \mid \frac{n h}{\operatorname{gcd}(n,|s|)} .
$$

and $s_{1}, h_{1}$ satisfy

$$
h_{1} \mid h, \quad \frac{h}{h_{1}} s_{1} \equiv s \bmod n \text {. }
$$


(ii) The values of every string $\left\{x_{i}^{t}\right\}$ in the limit cycle satisfy

$$
x_{i-s}=f^{h}\left(x_{i-r h}, \ldots, x_{i}, \ldots, x_{i+r h}\right),
$$

where $f^{h}$ represents the $h^{\text {th }}$ order composition of the rule. If (4.1) provides an explicit expression for some $x_{j}$ in terms of site values with smaller indices, then it is a recurrence relation for the values of any string for which the rule acts as a shift $s / h$. If the expression is implicit, a recurrence relation is obtained by rewriting (4.1), for each value of $n=1,2, \ldots$, as

$$
x_{l-s \pm n}=f^{h}\left(x_{i-r h}, \ldots, x_{i}, \ldots, x_{i+r h}\right) .
$$

Then a limit cycle with this shift exists on a cylinder size $n$ iff the above recurrence relation (either in form (4.1) or (4.2)) is satisfied by a sequence of (spatial) period $n$; i.e.,

$$
x_{i+n}=x_{i}, \text { for all } i .
$$

As a simple example illustrating the results of Theorem 3, consider the rule defined by

$$
\{01,10,11\} \rightarrow 0, \quad\{00\} \rightarrow 1
$$

or equivalently,

$$
f\left(x_{0}, x_{1}\right)=\overline{x_{0} x_{1}},
$$

where $\bar{x}$ denotes "not $x$." (Considered as a nearest-neighbor rule, (4.3) is labelled Rule 3 in the scheme of [4].) Before Theorem 3 can be invoked, it is necessary to establish the role of shifts in generating limit cycle behavior for this rule.

Proposition. Each primitive limit cycle of least period p for the rule (4.3) is generated by a shift $\sigma$ whose fundamental values are given by

$$
\begin{aligned}
\sigma & =\frac{p+1}{2}=\frac{1-p}{2}, \quad p \text { odd } \\
& =\frac{1}{2}, \quad p \text { even. }
\end{aligned}
$$

Proof. It will first be shown that the set of strings exhibiting such a shift, and the set of strings belonging to some primitive limit cycle of the rule, are equivalent.

Suppose a string has shift of (not necessarily fundamental) value $\sigma=\frac{1}{2}$. Then from Theorem 3(ii), it follows that the relation

$$
\begin{aligned}
x_{i+1} & =f^{2}\left(x_{i}, x_{i+1}, x_{i+2}\right) \\
& =f\left[\overline{x_{i} x_{i+1}}, \overline{x_{i+1} x_{i+2}}\right] \\
& =\left(x_{i} x_{i+1}+x_{i}+x_{i+1}\right)\left(x_{i+1} x_{i+2}+x_{i+1}+x_{i+2}\right) \\
& =x_{i} x_{i+1} x_{i+2}+x_{i} x_{i+2}+x_{i+1}
\end{aligned}
$$

must hold. The above can be rewritten as a relation for $x_{i+2}$ given arbitrary values of $x_{i}$ and $x_{i+1}$; i.e.,

$$
\begin{aligned}
x_{i+2} & =0, & \text { if } x_{i}=1 \text { and } x_{i+1}=0, \\
& =\{0 \cup 1\}, & \text { otherwise },
\end{aligned}
$$


where the " $\cup$ " indicates that either value may be used. It is easy to show that (4.5) is satisfied by any string of values in which the block 101 does not appear. Any such string of length $n$ therefore exhibits a shift size $\frac{1}{2}$, and belongs to a limit cycle of period $p \mid 2 n$. Conversely, and string containing 101 has no predecessor under the rule, and hence cannot belong to a limit cycle. Thus, every string in a limit cycle must have shift $\frac{1}{2}$.

A shift size $\frac{1}{2}$ cannot be fundamental, however, for a limit cycle of odd period length. Thus for $p$ odd, it must be true that the fundamental shift is given by $\sigma=s / h$, where $h=1$. Then it follows that $2 \sigma \equiv 1 \bmod n$, or $\sigma=(n+1) / 2$, where $n$ is the length of the cylinder, and $n$ must be odd. In this case,

$$
p=\frac{n}{\operatorname{gcd}\left(n, \frac{n+1}{2}\right)}=n
$$

Finally, if the period $p$ is even, then it follows from the above argument that $\sigma=\frac{1}{2}$ is fundamental, and thus the proposition has been proved.

Examples of conclusions that may be drawn on the basis of Theorem 3 and the above proposition include:

(a) The cylinder sizes $n$ on which there exists a primitive limit cycle such that the action of the rule $R$ is a shift of integer value $\sigma$ are given by the periods of the sequences generated, using arbitrary initial conditions, by the recurrence relation

$$
x_{i-\sigma}=f\left(x_{i-1}, x_{i}\right)
$$

where $f$ is defined by (4.3). For example, with $\sigma=-3$ and initial condition

$$
x_{0}=x_{1}=x_{3}=0, \quad x_{2}=1,
$$

the sequence generated by (4.6) is

00101000011 , (the - indicating that the sequence repeats itself),

of period 7. Moreover, given any other initial condition, the periodic sequence generated by (4.6) is (ultimately) the same. Hence the string 1000011 defined on a cylinder size $n=7$ is the unique primitive limit cycle for which the rule acts as a shift $\sigma=-3$. From Theorem 3(i), its period is given by

$$
p=\frac{n h}{\operatorname{gcd}(n,|s|)}=7 \text {. }
$$

(b) Consider the rule $R^{*}$ defined by $\{000,001,100\} \rightarrow 0,\{010,011,101,110,111\} \rightarrow 1$ (Rule 236 in the labelling scheme of [4]). Then

$$
R^{*}=\mu\left(R^{2}\right)
$$

where $\mu$ denotes the left-shift operator. Hence, given any string on which $R$ acts as a shift of size $\sigma, R^{*}$ acts as a shift size

$$
\sigma\left(R^{*}\right)=2 \sigma-1 \text {. }
$$


From (4.4) it follows that

$$
\sigma\left(R^{*}\right)=0
$$

for all limit cycles, and thus the only attractors for this rule are fixed points. Furthermore, on any cylinder size $n$, the number of primitive fixed points, denoted here by $\delta_{n}^{(1)}$, is given by

$$
\begin{aligned}
& \delta_{n}^{(1)}=L_{n}^{(n)}, \quad n \quad \text { odd }, \\
& =L_{n / 2}^{(n)}, n \text { even, }
\end{aligned}
$$

where $L_{n}^{(p)}$ denotes the number of limit cycles for $R$.

\section{Shifts as the Basic Mechanism for Periodic Behavior}

The preceding section, together with the Appendix, lays the groundwork for a theorem on the fundamental role of shifts in generating limit cycle behavior for all two-neighbor automata rules. The theorem uses the fundamental shift value associated with the limit cycle generated by the initial condition consisting of a single non-zero site on a cylinder size $n$ to define a relation that must be satisfied by any string of length $n$ that appears in a limit cycle for a given rule $R$.

In what follows, a two-neighbor rule is assumed to be either of the form

$$
x_{i}^{t+1}=f\left(x_{i-1}^{t}, x_{i}^{t}\right)
$$

or of the form

$$
x_{i}^{t+1}=f\left(x_{i}^{t}, x_{i+1}^{t}\right) .
$$

Theorem 4. Let $R$ be any two-neighbor automata rule defined by a function $f$, and let $\sigma_{n}=s / h$ be the fundamental shift associated with the limit cycle on a cylinder size $n$ generated by the evolution under $R$ of the initial condition consisting of a single non-zero site value. Then a string $S=\left\{x_{i}^{t}\right\}$ appears in a limit cycle for $R$ on a cylinder size $n$ iff its values satisfy the relation

$$
x_{i-s}=f^{h}\left(x_{i-h}, \ldots, x_{i}\right)
$$

in the case of a "left-handed" rule, or

$$
x_{i-s}=f^{h}\left(x_{i}, \ldots, x_{i+h}\right),
$$

in the case of a "right-handed" rule. If Eqs. $(5.1 a, b)$ represent recurrence relations, then all possible strings appearing in limit cycles for $R$ are generated by using all possible initial conditions for the appropriate relation. Otherwise, the strings are generated by rewriting the relations as

$$
x_{l-s \pm n}=f^{h}\left(x_{i-h}, \ldots, x_{i}\right)
$$

or as

$$
x_{i-s \pm n}=f^{h}\left(x_{i}, \ldots, x_{i+h}\right),
$$

and again using all possible initial conditions. The period $p$ of the limit cycle in which 
$S$ appears satisfies

$$
p \mid \frac{n h}{\operatorname{gcd}(n,|s|)} .
$$

Proof. The set of two-neighbor rules, along with the characterizations of their attractor structures, are listed in the Appendix. Given the results of Sect. 4, it is then straightforward to establish the following:

(a) Rules $0,12,207,68,221,192,252,130,238,204: \sigma_{n}=n$, and every attractor is a fixed point with shift $\sigma=n$;

(b) Rules $3,63,17,119: \sigma_{n}=\frac{1}{2}$, and every attractor is a limit cycle with shift value given by (4.4);

(c) Rules 15,85: $\sigma_{n}=\frac{2}{2}$, and attractors consist of limit cycles of odd least period $p$ with shift $\sigma=p+1$, and limit cycles of even least period with shift $\sigma=\frac{2}{2}$;

(d) Rules 48, 243, 34, 187, 240, 42: $\sigma_{n}=1$, and attractors consist of fixed points (strings of all 0's), with shift $\sigma=0$, and limit cycles with shift $\sigma=1$;

(e) Rules $60,195,102,153$ : The linearity of these rules implies that every limit cycle string on a cylinder size $n$ must satisfy the fundamental shift relation given by (5.1).

It therefore follows that every string that appears in a limit cycle for a rule $R$ on a cylinder size $n$ satisfies the appropriate relation (5.1).

Next show that the converse is true; i.e., that every sequence satisfying the appropriate relation (5.1) appears in a limit cycle for the rule $R$ on a cylinder of size $n$. It is necessary first to prove that the (spatial) period of every such sequence divides $n$. For cases (a) and (d), this result is easily established. For case (b), the appropriate recurrence relation is given by

$$
x_{i-1+n}=f^{2}\left(x_{i-2}, x_{i-1}, x_{i}\right)
$$

of order $n+1$. It is easy to show that any initial condition $\left\{y_{i}, i=0, \ldots, n-1\right\}$ in which "101" does not occur generates a solution $\Sigma$ of period $n$ with $x_{i}=y_{i+1}$, $i=0, \ldots, n-1$. Even if " 101 " does occur in the initial condition, it cannot survive in $\Sigma$ since $f^{2}(0,1,0)=f^{2}(0,1,1)=1$, and hence a new string $\Sigma$ of length $n$ in which " 101 " does not occur is generated. The period is therefore $n$.

Now consider case (c). The appropriate recurrence relation is given by

$$
x_{i-2+n}=f^{2}\left(x_{i-2}, x_{i-1}, x_{i}\right)
$$

of order $n$. Any initial condition $\left\{y_{i}, i=0, \ldots, n-1\right\}$ generates a solution $\Sigma=\left\{x_{i}\right\}$ of period $n$ consisting of concatenations of itself.

Lastly, consider case (e). Suppose $n$ is odd. It can be shown (see [6] for details) that the recurrence relation $F\left(x_{i}\right)$ associated with the fundamental shift is of the form

$$
F\left(x_{i}\right)=\sum_{j=0}^{j=n-1} x_{i+j}=0 .
$$

For the initial condition $x_{0}=\cdots=x_{n-3}=0, x_{n-2}=1$, it follows that $x_{n-1}=1$, and thus the infinite sequence $\Sigma$ generated by the recurrence relation is of period $n$. 
If $n=2^{m} q$, where $j$ is odd, then it is easy to show that

$$
F\left(x_{i}\right)=\sum_{j=0}^{j=q-1} x_{i+2^{m} j}=0,
$$

of order $N=2^{m}(q-1)+1$. For the initial condition $x_{0}=\cdots=x_{N-3}=0, x_{N-2}=1$, the recurrence relation implies that $x_{i}=1, i=n-1, n-1-2^{m}$, and $x_{i}=0$ for all other $0 \leqq i \leqq n-1$. As before, the infinite sequence generated by the relation is of period $n$.

Next show that every other sequence satisfying the relation has spatial period $n$. Let $\vec{x}_{i}$ denote the vector $\left(x_{i}, \ldots, x_{i+k-1}\right)$, where $k$ is the order of the recurrence relation. Use the initial conditions defined above (consisting of all zeros with the exception of $\left.x_{k-2}=1\right)$ to generate a sequence satisfying the relation. Then the vectors $\left\{\vec{x}_{0}, \ldots, \vec{x}_{k-1}\right\}$ form a basis for the $k$-dimensional space of all possible initial conditions for a $k^{\text {th }}$ order recurrence relation. Each vector in the basis represents an initial condition that generates an infinite sequence of period $n$. Since every other initial condition can be expressed in terms of the basis vectors, linearity of the rule implies that the period of any other solution of $F\left(x_{i}\right)=0$ must divide $n$.

Thus, it has been shown that the period of every solution of (5.1) (for all possible two-neighbor rules) divides $n$.

Finally, it is clear that any sequence satisfying the appropriate relation (5.1) undergoes a shift under the action of the automata rule $R$, and hence the sequence appears in a limit cycle for $R$.

\section{Discussion}

The main concepts introduced in this paper for the study of attractors for cylindrical cellular automata include:

(i) the definition of a matrix whose powers correspond to the cylinder sizes on which the rule is defined;

(ii) the derivation of linear recurrence relations for the number $T_{n}^{(p)}$ of strings appearing in a limit cycle of fixed period $p$ on a cylinder size $n$ in terms of $T_{j}^{(p)}$ with $j<n$;

(iii) the identification of shift mechanisms as key to understanding limit cycle behavior.

The introduction of the invariance matrix provides a technique for counting the number of fixed points, or more generally, limit cycles, for an automata rule. For a given period $p$, the technique determines all cylinder sizes on which a limit cycle of period $p$ can exist, and the number of limit cycles of period $p$ on each such cylinder size. The invariance matrix used here is the same as the connectivity matrix associated with a directed graph. In a connectivity matrix, the elements $a_{i j}$ provide a count of "admissible" paths beginning at node $i$ and ending at node $j$. For cellular automata, the nodes represent tuples of site values, and the paths can be taken to be spatial (rather than temporal, as might at first be assumed) sequences of these tuples. In this context, then, "admissible" means "invariant under the rule," and thus the matrix contains a " 1 " wherever a given value may be appended to a spatial 
sequence with invariance being preserved, and a " 0 " elsewhere. Clearly, admissibility may be defined in other ways, and the technique is thus helpful in other contexts as well. (In [11], for example, the technique is used to study preimages under cellular automata rules for spatial sequences.)

In the study of attractors for cellular automata on cylinders, the essential aspect of the invariance matrix $A$ is that the trace of powers of $A$ provides the number of fixed points on corresponding cylinder sizes. Equivalently, this number may be found by computing powers of the eigenvalues associated with $A$. These powers of eigenvalues correspond to terms in the characteristic equation of $A$. Thus, for arbitrary cellular automata rules, the number of strings belonging to limit cycles of fixed period on a cylinder size $n$ is given as a solution to a linear recurrence relation involving the number on smaller cylinders.

The study of cylindrical cellular automata indicates that shift mechanisms are key to understanding periodicity in these systems. A rule acts upon a string as a shift of size $\sigma=s / h$ if, under the evolution of the automaton, the string shifts $s$ sites in $h$ iterations. It has been shown that all limit cycles for all two-neighbor rules are generated by shifts in the sense that the values of any string in the limit cycle must satisfy a relation defined using a shift value depending on $n$. Limit cycle periods, cylinder sizes, and shifts are connected in well-defined ways, and an analysis of shifts provides detailed information otherwise not available on limit cycle behavior.

The fundamental role of shifts in more general cellular automata limit cycle behavior is conjectured, but not proved. Shift structures for linear rules of arbitrary neighborhood size are discussed in [6]. Examples-- such as that of Rule 30, a nonlinear nearest-neighbor rule that has been proposed as an efficient pseudorandom number generator [12] - indicate that nonlinear rules of arbitrary neighborhood size exhibit highly complicated shift structures.

The analysis of shifts in generating limit cycle behavior suggests an equivalence between the strings of values appearing in limit cycle for cylindrical cellular automata, and recurring sequences in finite fields. The latter is a rich and well-developed topic of interest both theoretically and practically. In [6], the implications of this equivalence are explored.

\section{Appendix: Attractors for Two-Neighbor Automata Rules}

This appendix provides characterizations for the set of attractors for two-neighbor automata rules. The results for $L_{n}^{(p)}$, the number of primitive limit cycles of least period $p$ on a cylinder size $n$, provide analytical expressions for the corresponding computational results contained in Table 14 of [4].

Notation. Let

(a) "*" be a "wild card" symbol for which either " 0 " or " 1 " may be substituted;

(b) $G(n)$ be the total number of primitive strings of length $n$;

(c) $C(A)$ be the characteristic equation of the matrix $A$ with the roots $\lambda=0$ not pearing;

(d) $Q[f(\lambda)]$ be the highest power of $\lambda$ appearing in an arbitrary function $f(\lambda)$; 
(e) $T^{(p)}=Q\left[C\left(A_{p}\right)\right]$ if $C\left(A_{p}\right)$ is of the form $\prod_{i}\left(\lambda^{m_{i}}-1\right)^{\alpha_{2}}$;

(f) $\left.q\right|^{*} p$ be any positive integer $q$ such that $q \mid p$ and $q \neq p$.

Remark 1. $G(n)$ satisfies the relation $G(n)=2^{n}-\sum_{m \mid *_{n}} G(m)$, with $G(1)=2$.

Remark 2. $T^{(p)}=\sum_{i} m_{i} \alpha_{i}$ represents the total number of primitive strings that belong to a limit cycle of (not necessarily least) period $p$.

The set of two-neighbor rules, defined as being either of the form

or

$$
x_{i}^{t+1}=f\left(x_{i-1}^{t}, x_{i}^{t}\right)
$$

$$
x_{i}^{t+1}=f\left(x_{i}^{t}, x_{i+1}^{t}\right),
$$

is grouped below according to equivalences under symmetry transformations. In each case, the rule numbers are given according to the scheme of [4], with the rules being considered, to avoid ambiguity in labelling, of neighborhood size 3 . The definitions of the rules correspond, in each equivalence class, to the first rule number listed.

I. Rules $(0,255)\{* 00, * 01, * 10, * 11\} \rightarrow 0$ :

(a) Characteristic equation:

$$
C\left(A_{p}\right)=(\lambda-1), \text { for all } p .
$$

(b) Number of primitive limit cycles:

$$
\begin{aligned}
& L_{1}^{(1)}=1, \\
& L_{n}^{(p)}=0, \quad \text { otherwise. }
\end{aligned}
$$

II. Rules $(3,17,63,119)\{01 *, 10 *, 11 *\} \rightarrow 0,\{00 *\} \rightarrow 1$ :

(a) Characteristic equation:

Using the notation

$$
\begin{aligned}
{[p] } & =p, \quad \text { if } p \text { odd, } \\
& =\frac{p}{2}, \quad \text { if } p \text { even, } \\
C\left(A_{p}\right) & =\left\{\operatorname{lcm}_{q !^{*} p} C\left(A_{q}\right)\right\}\left(\lambda^{[p]}-1\right)^{\alpha} . \\
\text { where } \alpha[p]+Q\left[\operatorname{lcm}_{\left.q\right|^{*} p} C\left(A_{q}\right)\right] & =T^{(p)} \text {, and } \\
T^{(p)} & =T^{(p-2)}+T^{(p-3)}, p>3,
\end{aligned}
$$

with $T^{(1)}=0, T^{(2)}=2, T^{(3)}=1$.

(b) Number of primitive limit cycles:

$$
\begin{aligned}
L_{n}^{(p)}=\alpha, & \text { if } n=[p], \\
& =0, \quad \text { otherwise, }
\end{aligned}
$$

where $\alpha,[p]$ are defined in (a). 
III. Rules $(12,68,207,221)\{00 *, 10 *, 11 *\} \rightarrow 0,\{01 *\} \rightarrow 1$ :

(a) Characteristic equation:

$$
C\left(A_{p}\right)=\lambda^{2}-\lambda-1, \text { for all } p .
$$

(b) Number of primitive limit cycles:

$$
L_{n}^{(1)}=L_{n-2}^{(1)}+L_{n-1}^{(1)},
$$

with $L_{1}^{(1)}=1$, and $L_{2}^{(1)}=3$,

$$
L_{n}^{(p)}=0, \quad p \neq 1 .
$$

IV. Rules $(15,85)\{10 *, 11 *\} \rightarrow 0,\{00 *, 01 *\} \rightarrow 1:$

(a) Characteristic equation:

For $p$ odd,

$$
C\left(A_{p}\right)=\left\{\operatorname{lcm}_{\left.q\right|^{*} p} C\left(A_{q}\right)\right\}\left(\lambda^{2 p}-1\right)^{\alpha},
$$

where $2 \alpha_{p} p=G(p)$.

For $p=2 k, k$ odd,

$$
C\left(A_{p}\right)=\left\{\operatorname{lcm}_{\left.q\right|_{p} ^{*}} C\left(A_{q}\right)\right\}\left(\lambda^{p}-1\right)^{\beta_{1}}\left(\lambda^{k}-1\right)^{\beta_{2}},
$$

where

$$
\begin{aligned}
\left(\beta_{1}+\alpha_{k}\right) p & =G(p), \\
\beta_{2} k & =G(k),
\end{aligned}
$$

where $\alpha_{k}$ is defined above.

For $p=2 k$, $k$ even,

with

$$
C\left(A_{p}\right)=\left\{\operatorname{lcm}_{\left.q\right|^{*}} C\left(A_{q}\right)\right\}\left(\lambda^{p}-1\right)^{\beta},
$$

$$
\beta p=G(p)
$$

(b) Number of primitive limit cycles:

For $p$ odd,

$$
\begin{aligned}
L_{n}^{(p)} & =2 \alpha_{p}, & & n=2 p \\
& =0, & & \text { otherwise }
\end{aligned}
$$

For $p=2 k, k$ odd,

$$
\begin{aligned}
L_{n}^{(p)} & =\frac{\beta_{2}}{2}, & & n=k, \\
& =\beta_{1}, & & n=p, \\
& =0, & & \text { otherwise. }
\end{aligned}
$$

For $p=2 k, k$ even, 


$$
\begin{aligned}
L_{n}^{(p)} & =\beta, & & n=p \\
& =0, & & \text { otherwise }
\end{aligned}
$$

where $\alpha_{p}, \beta_{1}, \beta_{2}, \beta$ are defined in (a).

V. Rules $(34,48,187,243)\{* 00, * 10, * 11\} \rightarrow 0,\{* 01\} \rightarrow 1$ :

(a) Characteristic equation:

$$
C\left(A_{p}\right)=\left\{\underset{\left.q\right|^{*}}{1 \mathrm{~cm}} C\left(A_{q}\right)\right\}\left(\lambda^{p}-1\right)^{\alpha},
$$

where $\alpha p+Q\left[\underset{\left.q\right|^{*} p}{\operatorname{lcm}} C\left(A_{q}\right)\right]=T^{(p)}$, and

$$
T^{(p)}=T^{(p-1)}+T^{(p-2)}, \quad p>2,
$$

with $T^{(1)}=1, T^{(2)}=3$.

(b) Number of primitive limit cycles:

$$
\begin{aligned}
& L_{n}^{(p)}=\alpha, \text { for } n=p, \\
& =0, \quad \text { otherwise, }
\end{aligned}
$$

where $\alpha$ is defined in (a).

VI. Rule (51) $\{01 *, 11 *\} \rightarrow 0,\{00 *, 10 *\} \rightarrow 1$ :

(a) Characteristic equation:

$$
\begin{aligned}
C\left(A_{p}\right) & =\lambda^{2}, & & p \text { odd } \\
& =\lambda-2, & & p \text { even } .
\end{aligned}
$$

(b) Number of primitive limit cycles:

$$
\begin{aligned}
L_{n}^{(p)} & =G(n), & & p=2, \\
& =0, & & \text { otherwise. }
\end{aligned}
$$

VII. Rules $(60,102,153,195)\{00 *, 11 *\} \rightarrow 0,\{01 *, 10 *\} \rightarrow 1$ :

(a) Characteristic equation:

With every recurrence relation $\sum a_{j} x_{j}$, associate a polynomial $\sum a_{j} x^{j}$.

Then

$$
C\left(A_{p}\right)=\left\{\begin{array}{l}
\operatorname{lcm} C\left(A_{q}\right) \\
\left.q\right|^{*} p
\end{array} \prod_{i}\left(\lambda^{m_{t}}-1\right)^{\alpha_{i}},\right.
$$

where each $m_{i}$ is the spatial least period of an infinite sequence satisfying the recurrence relation corresponding to

$$
\frac{(x+1)^{p}-1}{\operatorname{lcm}_{\left.q\right|^{*} p}\left[(x+1)^{q}-1\right]}
$$

and $\alpha_{i}$ is the number of distinct such sequences. (See [6] for further details.)

(b) Number of primitive limit cycles: 


$$
\begin{aligned}
L_{n}^{(p)} & =\frac{\alpha_{i}}{p}, & & n=m_{i}, \\
& =0, & & \text { otherwise, }
\end{aligned}
$$

where $\alpha_{i}, m_{i}$ are defined in (a).

VIII. Rules $(136,192,238,252)\{* 00, * 01, * 10\} \rightarrow 0,\{* 11\} \rightarrow 1$ :

(a) Characteristic equation:

$$
C\left(A_{p}\right)=(\lambda-1)^{2}, \text { for all } p,
$$

(b) Number of primitive limit cycles:

$$
\begin{aligned}
& L_{1}^{(1)}=2, \\
& L_{n}^{(p)}=0, \quad \text { otherwise. }
\end{aligned}
$$

IX. Rule (204) $\{00 *, 10 *\} \rightarrow 0,\{01 *, 11 *\} \rightarrow 1$ :

(a) Characteristic equation:

$$
C\left(A_{p}\right)=(\lambda-2), \text { for all } p .
$$

(b) Number of primitive limit cycles:

$$
\begin{aligned}
L_{n}^{(p)} & =G(n), & & p=1, \\
& =0, & & \text { otherwise. }
\end{aligned}
$$

X. Rules $(170,240)\{* 00, * 10\} \rightarrow 0,\{* 01, * 11\} \rightarrow 1$ :

(a) Characteristic equation:

$$
C\left(A_{p}\right)=\left\{\lim _{\left.q\right|^{*} p} C\left(A_{q}\right)\right\}\left(\hat{\lambda}^{p}-1\right)^{\alpha},
$$

where $\alpha p=G(p)$.

(b) Number of primitive limit cycles:

$$
\begin{aligned}
& L_{n}^{(p)}=\alpha, \quad \text { for } n=p, \\
& =0, \quad \text { otherwise, }
\end{aligned}
$$

where $\alpha$ is defined in (a).

Acknowledgements. This work was supported in part by the NSF under Grant No. DMS-8601520 and by the DOE Office of Scientific Computing under Contract No. KC-07-01-01.

\section{References}

1. Martin, O., Odlyzko, A., Wolfram. S.: Algebiarc propertes of cellular automata. Commun. Math. Phys. 93, 219-259 (1984)

2. Guan, P., He, Y.: Exact results for deterministic cellular automata. J. Stat. Phys. 43, 463 (1986)

3. Guan, P., He, Y.: Upper bound on the number of cycles in border-decisive cellular automata. Complex Systems 1, 181 (1987)

4. Wolfram, S.: Theory and applications of cellular automata. Singapore: World Scientific 1986

5. Jen, E.: Global properties of cellular automata. J. Stat. Phys. 43, 219 (1986) 
6. Jen, E.: Linear cellular automata and recurring sequences in finite fields. Los Alamos National Laboratory Report LA-UR-87-2026. Commun. Math. Phys. (to appear)

7. Jen, E.: Invariant strings and pattern-recognizing properties of one-dimensional cellular automata. J. Stat. Phys, 43, 243 (1986)

8. Hedlund, G.: Endomorphisms and automorphisms of the shift dynamical system. Math. Syst. Theory 3, 320 (1969)

9. Lind, D. A.: Applications of ergodic theory and sofic systems to cellular automata in reference [1]

10. Boyle, M.: Constraints on the degree of a sofic homomorphism and the induced multiplication of measures on unstable sets. Israel J. Math. 53, 52 (1986)

11. Jen, E.: Scaling of preimages in cellular automata. Complex Systems 6, 1045-1062 (1987)

12. Wolfram, S.: Random sequence generation by cellular automata. Adv. Appl. Math. 7, 123 (1986)

Communicated by J. L. Lebowitz

Received August 7, 1987; in revised form February 9, 1988 